\title{
The benefits of dual and double ovulatory triggers in assisted reproduction
}

\author{
Rony T. Elias $^{1} \cdot$ Nigel Pereira ${ }^{1}$ (D) $\cdot$ Gianpiero D. Palermo $^{1}$
}

Received: 7 June 2017 / Accepted: 13 June 2017 / Published online: 21 June 2017

(C) Springer Science+Business Media, LLC 2017

We thank Dr. Orvieto for his interest in our recently published article [1] reporting the utility of a combined gonadotropinreleasing hormone agonist (GnRH-a) and human chorionic gonadotropin ( $\mathrm{hCG}$ ) ovulatory trigger in improving intracytoplasmic sperm injection (ICSI) cycle outcomes in patients with a history of poor fertilization in prior ICSI cycles with standard hCG trigger alone. The benefits of such combined or 'dual' triggers are being increasingly harnessed to improve the efficiency of donor oocyte-recipient cycles, elective oocyte cryopreservation, or fertility preservation prior to cancer therapy. It is also enthusing to note the increasing scope of dual triggers in challenging scenarios such as poor fertilization, abnormal follicular maturation and poor oocyte maturity $[2,3]$. We are grateful for Dr. Orvieto's timely letter that discusses additional indications for the administration of a dual trigger. Although prolongation of the time interval between the ovulatory trigger and oocyte retrieval has been described in the setting of a standard hCG trigger [4, 5], the modification of the dual trigger into a 'double trigger' by prolonging the time between ovulation triggering and oocyte retrieval seems very promising $[6,7]$. The benefits of dual and double ovulatory triggers accrued from the work of Dr. Orvieto as well as others will continue to improve the care of couples undergoing assisted reproduction.

Rony T. Elias

rta9002@med.cornell.edu

1 The Ronald O. Perelman and Claudia Cohen Center for Reproductive Medicine, Weill Cornell Medicine, 1305 York Ave, New York, NY 10021, USA
Compliance with ethical standards

Conflict of interest The authors declare that they have no conflict of interest.

\section{References}

1. Elias RT, Pereira N, Artusa L, Kelly AG, Pasternak M, Lekovich JP, et al. Combined GnRH-agonist and human chorionic gonadotropin trigger improves ICSI cycle outcomes in patients with history of poor fertilization. J Assist Reprod Genet. 2017;34(6):781-8.

2. Griffin D, Feinn R, Engmann L, Nulsen J, Budinetz T, Benadiva C. Dual trigger with gonadotropin-releasing hormone agonist and standard dose human chorionic gonadotropin to improve oocyte maturity rates. Fertil Steril. 2014;102(2):405-9.

3. Pereira N, Elias RT, Neri QV, Gerber RS, Lekovich JP, Palermo GD, et al. Adjuvant gonadotrophin-releasing hormone agonist trigger with human chorionic gonadotrophin to enhance ooplasmic maturity. Reprod BioMed Online. 2016;33(5):568-74.

4. Raziel A, Schachter M, Strassburger D, Kasterstein E, Ron-El R, Friedler S. In vivo maturation of oocytes by extending the interval between human chorionic gonadotropin administration and oocyte retrieval. Fertil Steril. 2006;86(3):583-7.

5. Pereira N, Neri QV, Lekovich JP, Palermo GD, Rosenwaks Z. The role of in-vivo and in-vitro maturation time on ooplasmic dysmaturity. Reprod BioMed Online. 2016;32(4):401-6.

6. Zilberberg E, Haas J, Dar S, Kedem A, Machtinger R, Orvieto R. Co-administration of GnRH-agonist and hCG, for final oocyte maturation (double trigger), in patients with low proportion of mature oocytes. Gynecol Endocrinol. 2015;31(2):145-7.

7. Haas J, Zilberberg E, Dar S, Kedem A, Machtinger R, Orvieto R. Coadministration of GnRH-agonist and hCG for final oocyte maturation (double trigger) in patients with low number of oocytes retrieved per number of preovulatory follicles - a preliminary report. J Ovarian Res. 2014;7:77. 